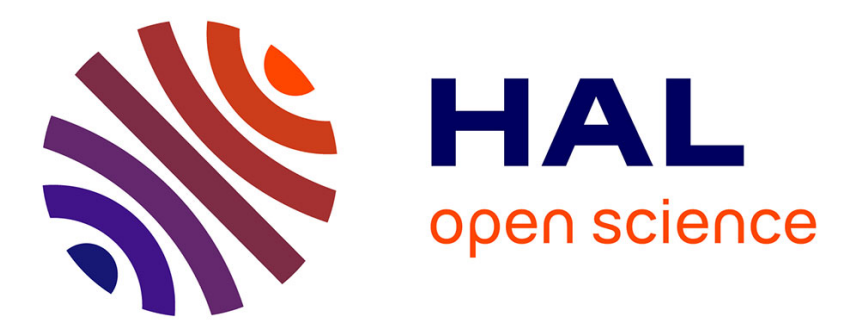

\title{
A novel device for intraoperative photodynamic therapy dedicated to glioblastoma treatment
}

Clément Dupont, Serge R. Mordon, Pascal Deleporte, Nicolas Reyns, Maximilien Vermandel

\section{- To cite this version:}

Clément Dupont, Serge R. Mordon, Pascal Deleporte, Nicolas Reyns, Maximilien Vermandel. A novel device for intraoperative photodynamic therapy dedicated to glioblastoma treatment. Future Oncology, 2017, Epub ahead of print. 10.2217/fon-2017-0261 . inserm-01611670

\section{HAL Id: inserm-01611670 https://www.hal.inserm.fr/inserm-01611670}

Submitted on 6 Oct 2017

HAL is a multi-disciplinary open access archive for the deposit and dissemination of scientific research documents, whether they are published or not. The documents may come from teaching and research institutions in France or abroad, or from public or private research centers.
L'archive ouverte pluridisciplinaire HAL, est destinée au dépôt et à la diffusion de documents scientifiques de niveau recherche, publiés ou non, émanant des établissements d'enseignement et de recherche français ou étrangers, des laboratoires publics ou privés. 


\title{
A novel device for intraoperative photodynamic therapy dedicated to glioblastoma treatment
}

\author{
C. Dupont, MSc, S. Mordon, PhD., P. Deleporte, MSc, N. Reyns, MD, PhD, M. \\ Vermandel $^{*}, \mathrm{PhD}$
}

Univ. Lille, Inserm, CHU Lille, U1189 - ONCO-THAI - Image Assisted Laser Therapy for Oncology, F-59000 Lille, France

*Corresponding author: m-vermandel@chru-lille.fr

\begin{abstract}
:
Introduction: Photodynamic therapy (PDT) appears to be a valuable new treatment modality for cancer therapy. Studies have reported the successful application of PDT for glioblastoma. Here, we introduce a new device dedicated to intraoperative PDT delivered early after fluoroguided resection combined with a transfer function that determines the treatment time based on the size of the surgical resection cavity.
\end{abstract}

Methods: First, we describe the device, which is composed of a trocar, a balloon filled with a diffusing solution, and a fiber guide in which a cylindrical light diffuser is inserted. Ex vivo experiments were performed to measure the fluence rate inside biological tissues. A calibration factor was defined to convert power measurements into fluence rate values. Calf brains were used to simulate light propagation in human brain tissue, and the photosensitizer administration effect on optical properties was discussed. The temperature elevation during illumination was evaluated.

Results: Light power was measured in tissues surrounding the device during ex vivo experiments. Using the previously characterized calibration factor, power measurements were converted to fluence rate values to obtain the transfer function. No thermal elevation was observed during a two-hour temperature test, and the impact of protoporphyrin IX on brain optical properties was considered negligible.

Conclusions: A discussion of experimental precision is presented. The light duration determined by the abacus had a standard deviation of less than one minute. This value is weak compared with the total illumination time necessary to treat one patient. The main advantage of our device lies in its straightforward implementation of intraoperative PDT for neurosurgery with acceptable dosimetry and easy treatment time.

Keywords: intraoperative PDT, instrumentation, fluoro-guided resection, glioblastoma, neurosurgery, medical device 


\section{Introduction}

Glioblastoma (GBM) is the most aggressive and common primary brain tumor. Its poor prognosis (median overall survival of 14.5 months) and incidence (approximately 5 new cases each year per 100,000 individuals) strongly encourage new adjuvant treatments [1]. Currently, photodynamic therapy (PDT) appears to be a valuable new treatment modality to improve the therapy of cancers at different locations [2]. In particular, many studies have reported that 5-aminolevulinic acid (5-ALA) PDT treats GBM [3-6]. Direct (apoptosis and tumor tissue necrosis) and indirect (angiogenesis inhibitor effect and immune response) effects contribute to the long-term destruction of the tumor.

According to the standard management of GBM [7], maximum tumor resection is expected when achievable. To improve the extent of resection (EOR), fluoro-guided resection (FGR) has recently been proposed [8]. Gliolan ${ }^{\circledR}$ (5-ALA) is administered to the patient before surgery. 5-ALA is a precursor of protoporphyrin IX (PpIX), a photosensitizer (PS) that preferentially accumulates in GBM cells. The PS is activated by a light emission wavelength matching the highest PS absorption peak. Thus, under blue light (400-410 nm), GBM cells shine pink-purple. Surgery is followed by radiation therapy and concomitant and adjuvant chemotherapy with the aim of slowing down recurrence. Nevertheless, relapse occurs in the cavity margin in $85 \%$ of cases [9], justifying the improvement of local control by a maximal EOR and additional therapy delivered intraoperatively [10]. In this context, intraoperative PDT may be applicable for treating areas bordering the resection cavity. However, illumination of the cavity remains an issue since the device must be suitable for the shape of the cavity, and in that context, a deformable balloon is expected to meet this requirement.

Previous studies have reported such a device for intraoperative GBM PDT. The first study conducted by Wilson et al. in 1986 [11] led to the creation of an irradiator able to replace the original volume of the removed tumor tissue. The device consisted of a balloon created from a glove middle finger and a stainless steel tube. The balloon was then filled with a diffusing solution (lipid emulsion (Abbott Laboratories Ltd, Chicago, IL, USA) concentrated at $0.1 \%$ ). The balloon was dilated from a diameter of 3 to $5 \mathrm{~cm}$. The optical fiber used was a frontal diffuser with a nominal numerical aperture of 0.4 coupled to a $7.5 \mathrm{~W}$ argon laser. The entire device was sterilized by heat. This irradiator was used in a phase I trial in which 9 patients were enrolled, with 4 having GBM. The administered PS was Photofrin (Photofrin Medical Inc.) at a dose of $2-2.5 \mathrm{mg} \cdot \mathrm{kg}^{-1}$. The study concluded with the intention to optimize their device, including the use of a cylindrical diffuser as the source.

In 2000, experiments with diffusing balloons reappeared by Dwyer et al. [12]. Their device was based on a laboratory-made balloon and a flexible nylon tube to handle the device during the surgery and carry the fiber. The balloon was filled with a saline solution. However, the silicone balloon was produced to induce reflections to homogenize the light distribution around the balloon. The optical fiber used was also a frontal diffuser coupled to a $3.5 \mathrm{~W}$ argon-ion laser. The device was cleaned with alcohol and gas sterilized. After 45 minutes of illumination at $3.5 \mathrm{~W}$, a temperature of $51^{\circ} \mathrm{C}$ was measured. The authors claimed that this temperature did not create thermal damage to the tissues. However, their experiments were conducted with a 1-1.5 W power output. This device was not used in a clinical trial. The objective of this study was to demonstrate the ability to produce devices with different shapes 
that best fit the shape of a resection cavity. This study was not dedicated to GBM treatment or to brain intraoperative surgery.

To our knowledge, the latest study dedicated to PDT for GBM with a balloon was reported by Moseley et al. [13, 14] in 2007. The device was created from a catheter balloon with a fiber terminating in a cylindrical diffuser coupled to a Diomed PDT laser at $630 \mathrm{~nm}$. The power emitted was not reported. Different liquids were tested to fill the balloon. A diffusing solution based on a lipid emulsion was reported to be the most suitable for increasing the scattering diffusion. Two different balloon sizes (diameters of $1.5 \mathrm{~cm}$ and 2.5 $\mathrm{cm}$ ) and different shapes (spherical and prolate spheroid) were studied. A small difference was measured between the two shapes. Different lengths of a cylindrical diffuser were used $(1.2 \mathrm{~cm}, 2.5 \mathrm{~cm}$ and $5 \mathrm{~cm})$. A $2.5 \mathrm{~cm}$ cylindrical diffuser length seemed to be more suitable for the spherical device, while the $5 \mathrm{~cm}$ one seemed to be more suitable for the prolate spheroid device. The authors concluded that the length of the cylindrical diffuser shall be the same as the device length. These devices were not used in a clinical trial but were steered toward brain surgery applications.

In this study, we describe a new lighting device dedicated to intraoperative GBM photodynamic treatment [15]. PDT is applied early after FGR where the 5-ALA is already used as a diagnostic agent under blue light. Once this lighting device is inserted into the cavity, red light at a sufficient fluence rate is expected to activate the 5-ALA accumulated within remaining tumor cells and to induce a PDT effect. Dosimetry issues because of interindividual variability of the surgical cavity shape were addressed by introducing a transfer function that easily allows evaluation of treatment duration.

In the following sections, we first introduce a methodology used to convert power measurements to fluence rate values with the help of a calibration factor and then describe ex vivo experiments performed to estimate the fluence rate in the tissue around the device. A temperature test was also conducted to evaluate thermal elevation.

\section{Material and methods}

\section{Material}

The device consisted of two parts (see figure 1). The first part is a balloon coupled to a trocar, commercialized by Aesculap ${ }^{\circledR}$ (Tuttlingen, Germany) and initially used in endoscopic operations in which tissue needs to be separated in the extraperitoneal space. This device is built from a sterilizable trocar body and a single-use only transparent balloon. A sluice gate is added to the trocar, allowing fluid insertion to inflate the balloon. The inflation of the balloon ranges from a $4 \mathrm{~cm}$ diameter for $40 \mathrm{~mL}$ of diffusing solution to a $9 \mathrm{~cm}$ diameter for $500 \mathrm{~mL}$ of injected diffusing solution. A silicone valve terminates the trocar to ensure permeability and pressure inside the device. 

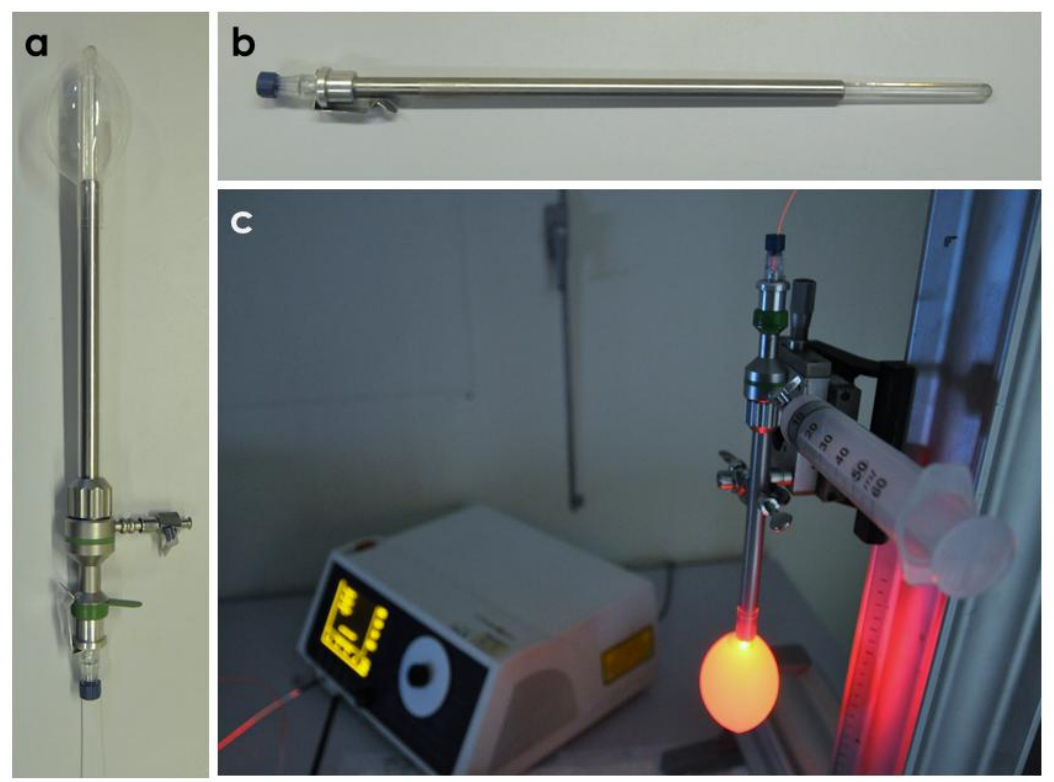

Figure 1 (a) Picture of the commercialized balloon with the addition of our fiber guide. (b) Picture of the optical fiber guide developed in the laboratory. (c) The whole device fixed on the optical bench.

The second part, referred to as the optical fiber guide, was developed in our laboratory. This guide was built from an $8 \mathrm{~mm}$ diameter borosilicate glass tube closed at one end and inserted in a $10 \mathrm{~mm}$ stainless steel tube (see figure 2). This guide enables positioning of the optical fiber at the center of the balloon while ensuring complete permeability. The optical fiber used is a $70 \mathrm{~mm}$ long cylindrical diffuser (RD-ML 70, Medlight, Ecublens, Switzerland) that approximately matches the balloon length in its deflated state. Finally, a screw thread fixed at the top of the guide allows tightening of the optical fiber inside the guide. The fiber guide is entirely compatible with the ethylene oxide sterilization process. 


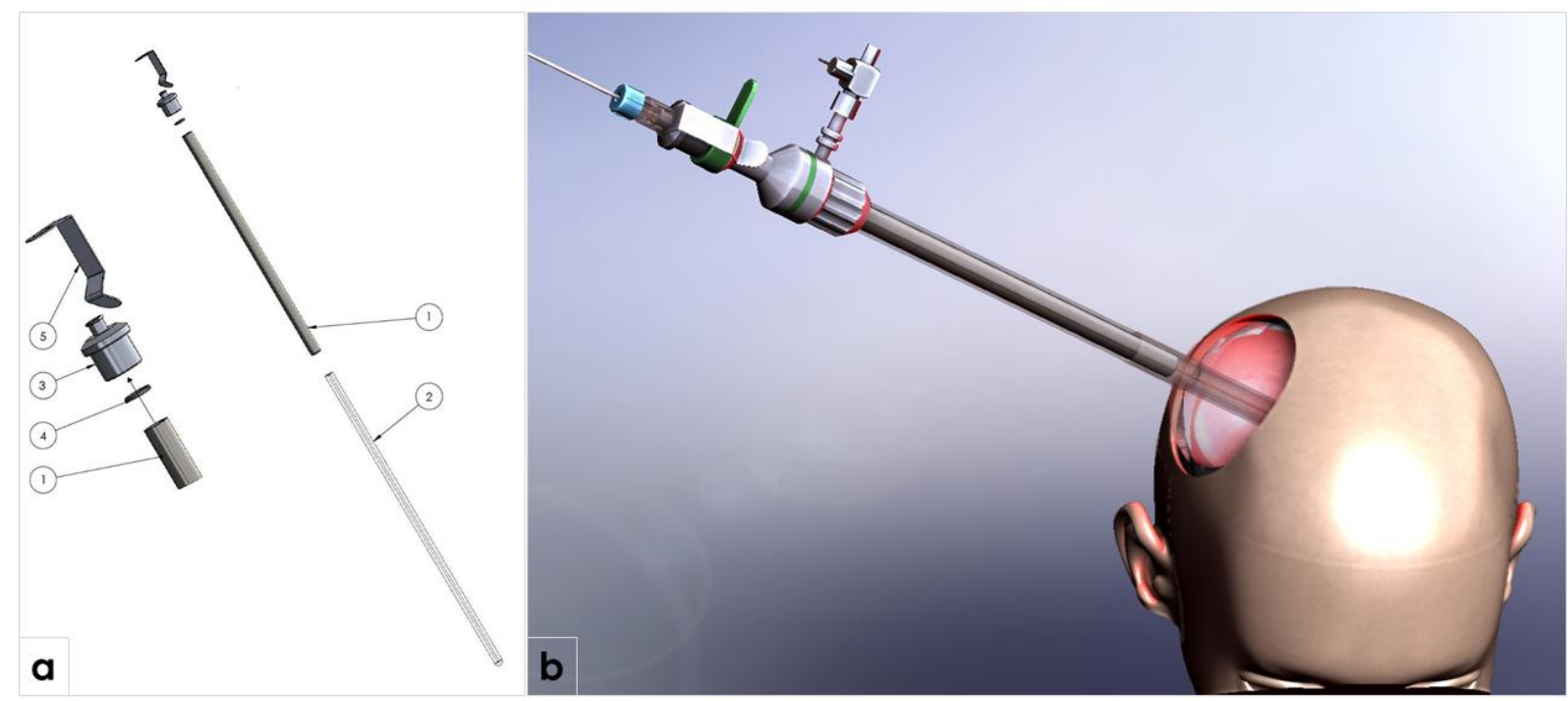

Figure 2 (a) Expanded view of the fiber guide: a borosilicate glass tube (2) is inserted into the stainless steel tube (1); a stainless steel luer adaptor (3), a PTFE seal for impermeability (5) and a stainless steel spring tab (5) hold the fiber guide against the trocar. (b) Perspective view (generated by SolidWorks ${ }^{\circledR}$ ) of the whole system: the fiber guide and the single-use balloon are inserted into the trocar; the whole device is placed into a surgical cavity.

To set up the whole lighting applicator, a single-use balloon is first inserted into the trocar. Then, the fiber guide is placed in the trocar balloon. After being assembled, the device is set on a frame and guided inside the cavity of the patient. The balloon is filled with an emulsion composed of a 20\% Intralipid (Fresenius Kabi France, Sèvres, France) diluted at a concentration of $0.1 \%$. This emulsion exhibits weak absorption and strong diffusion of 635 $\mathrm{nm}$ wavelength laser light $[11,13]$ and enables increased homogeneity of the light emitted from the source. Injection of diffusing solution into the balloon ends when the balloon wall reaches the brain-patient boundaries. This quantity of diffusing solution is the input value in our empirical dosimetry model that allows us to estimate the treatment duration. Indeed, a transfer function was modeled to estimate the light duration from the volume of diffusing solution injected to inflate the balloon within the surgical cavity. Finally, the $70 \mathrm{~mm}$ long cylindrical diffuser is inserted into the fiber guide.

\section{Ex vivo experiments}

The main purpose of the ex vivo study was to model a transfer function in such a manner that the treatment duration could be predicted from an abacus according to the quantity of diffusing solution injected into the balloon. The treatment time, deduced from the abacus, is expected to lead to a therapeutic fluence of $25 \mathrm{~J} / \mathrm{cm}^{2}$ at $5 \mathrm{~mm}$ inside surrounding brain tissues. The device was characterized with the same intraoperative PDT clinical conditions. The propagation of the light emitted from the balloon was quantified using several 
ex vivo experiments. To mimic clinical treatment conditions, calf brains were used due to their optical properties (absorption and reduced scattering coefficients of $0.19 \mathrm{~cm}^{-1}$ and 20.09 $\mathrm{cm}^{-1}$, respectively [16]) being close to those of the human brain (absorption and reduced scattering coefficients of $0.20 \mathrm{~cm}^{-1}$ and $20 \mathrm{~cm}^{-1}$, respectively) [3, 17, 18].

\section{Methods}

All experiments were performed using a $635 \mathrm{~nm} 3 \mathrm{~W}$ Ceralas laser (Biolitec, Jena, Germany). However, an input power of $2 \mathrm{~W}$ was used, which corresponds to the admissible limit power of the cylindrical diffuser.

Fluence rate values inside brain tissues around the balloon were measured to characterize our lighting device and to obtain the transfer function. Thus, we measured the light power propagation in tissues using an isotropic probe (IP85, Medlight, Ecublens, Switzerland) connected to a power meter to measure the power (1918-R, Newport, Irvine, United States) $10 \mathrm{~mm}$ around the balloon. The isotropic probe had to be calibrated to convert measured power values $\left(\mathrm{P}_{\text {measured }}, \mathrm{W}\right)$ to the fluence rate values in biological media $\left(\Psi_{\text {media, }}\right.$ $\left.\mathrm{W} / \mathrm{cm}^{2}\right)$.

\section{i. Calibration factor in air}

A calibration factor in air $\left(\mathrm{CF}_{\text {air }}\right)$ was computed to convert the power measurement to the irradiance in air. First, a frontal diffuser was used to create a uniform light beam (flat-top profile). A flat sensor (818-SL, Newport) with a sensitive area of $1 \mathrm{~cm}^{2}$ was placed in front of the uniform light beam at a distance $d$, allowing us to obtain a homogenous beam inside the sensing area. At the same distance, an isotropic probe was fixed on a rotating support. The spherical tip of the isotropic probe was irradiated by the same uniform light beam under the same experimental conditions.

Power was measured from both the flat sensor and from the isotropic probe according to the incidence angle $\mathrm{e}$ between the isotropic probe and the beam's direction (see figure 3 ). Angles where no data appear correspond to the distal part of the fiber (e.g., the part that was set up on the experimental bench support). Furthermore, as the support allowed only a $180^{\circ}$ rotation, the remaining $90^{\circ}$ were deduced via symmetry considerations to obtain the expected $270^{\circ}$ of the profile of the studied probe. Because the light beam is homogeneous and the air interaction with the light propagation at a short distance is negligible, the power measured with the flat sensor can be considered an irradiance measurement $\left(\mathrm{W} / \mathrm{m}^{2}\right)$.

Thus, the ratio between the irradiance measured with the flat sensor and the mean power measured with the isotropic sensor gave the calibration factor in air. However, this calibration factor in air is sensor dependent. 


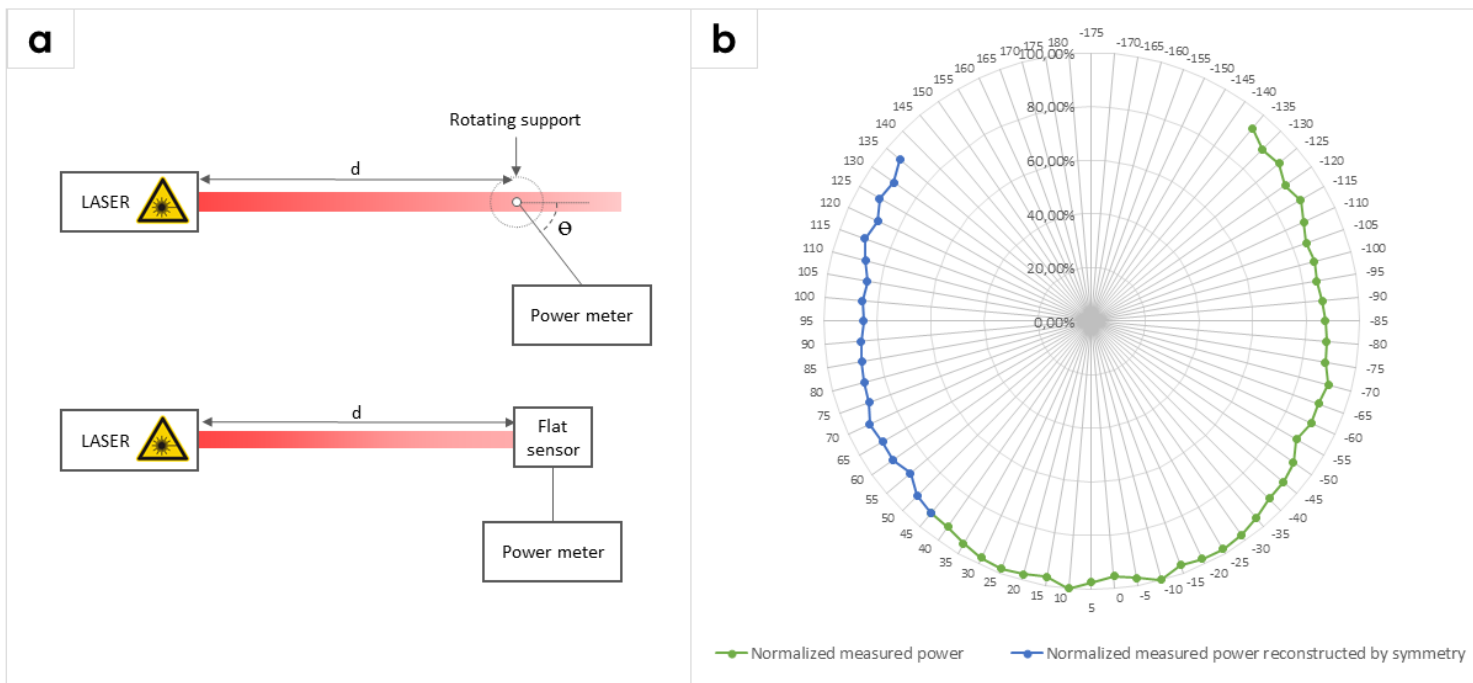

Figure 3 (a) Experimental setup used to compute the calibration factor in air: a flat sensor and an isotropic probe were placed in front of a uniform light beam. The isotropic probe was set on a rotary support to estimate the non-homogeneity response according to the irradiation angle. (b) Diagram showing the normalized power measured (in $\mathrm{nW}$ ) according to the incident angle between the probe and the laser beam. The blue part was reconstructed via symmetry considerations to obtain the entire measured power profile of the probe studied.

\section{ii. Calibration factor in brain tissue}

Once this factor was determined, we applied the Marijnissen and Star method [19,20] to convert this value in air to a calibration factor that was medium dependent $\left(\mathrm{FC}_{\text {media }}\right)$, thus converting the irradiance into a fluence rate. To obtain this correction, the following equation had to be solved:

$\Psi_{\text {media }}=\Psi_{\text {air }} \cdot \mathrm{F}_{\mathrm{n}} \cdot \mathrm{F}_{\mathrm{p}} \cdot \mathrm{F}_{\mathrm{b}} \cdot \mathrm{F}_{\mathrm{i}}=\left(\mathrm{P}_{\text {measured }} \cdot \mathrm{CF}_{\mathrm{air}}\right) \cdot \mathrm{F}_{\mathrm{n}} \cdot \mathrm{F}_{\mathrm{p}} \cdot \mathrm{F}_{\mathrm{b}} \cdot \mathrm{F}_{\mathrm{i}}$

where

$\mathrm{CF}_{\text {air }}$ : calibration factor in air $\left(1 / \mathrm{m}^{2}\right)$

$\mathrm{P}_{\text {measured }}$ : measured power of the biological tissue (W)

$\Psi_{\text {air }}$ : estimation of the irradiance in air $\left(\mathrm{W} / \mathrm{m}^{2}\right)$

$\Psi_{\text {media }}$ : estimation of the fluence rate in the biological medium $\left(\mathrm{W} / \mathrm{m}^{2}\right)$

$\mathrm{F}_{\mathrm{n}}$ : correction factor associated with the refractive index of the medium around the isotropic probe (dimensionless)

$\mathrm{F}_{\mathrm{p}}$ : perturbation correction (dimensionless)

$\mathrm{F}_{\mathrm{b}}$ : correction of the collection photon loss due to the surface occupied by the fiber on the detection sphere (dimensionless)

$\mathrm{F}_{\mathrm{i}}$ : correction of the sensor non-homogeneity response (dimensionless) 
Combining these empirical and theoretical factors resulted in a calibration factor that is expected to convert a power measurement into a fluence rate value for a given biological medium. Thus, with reliable fluence rate values, a treatment time can be estimated for a given fluence value.

\section{Experimental protocol}

Prior to the experiments, brains were prepared by dissecting out the cerebellum, the brain stem and the corpus callosum to improve the homogeneity of the studied tissues. We placed the device inside a black box to avoid reflections and then we surrounded the balloon with several pieces of calf brain (see figure 4). The isotropic probe was inserted into the medium until the probe touched the balloon wall. A first measurement was performed against the balloon wall. Then, the probe was pulled upward in $1 \mathrm{~mm}$ steps until the probe was 10 $\mathrm{mm}$ from the balloon wall. These measurements were repeated at 15 different positions around the balloon to decrease the variability due to the strong heterogeneity of the tissues.

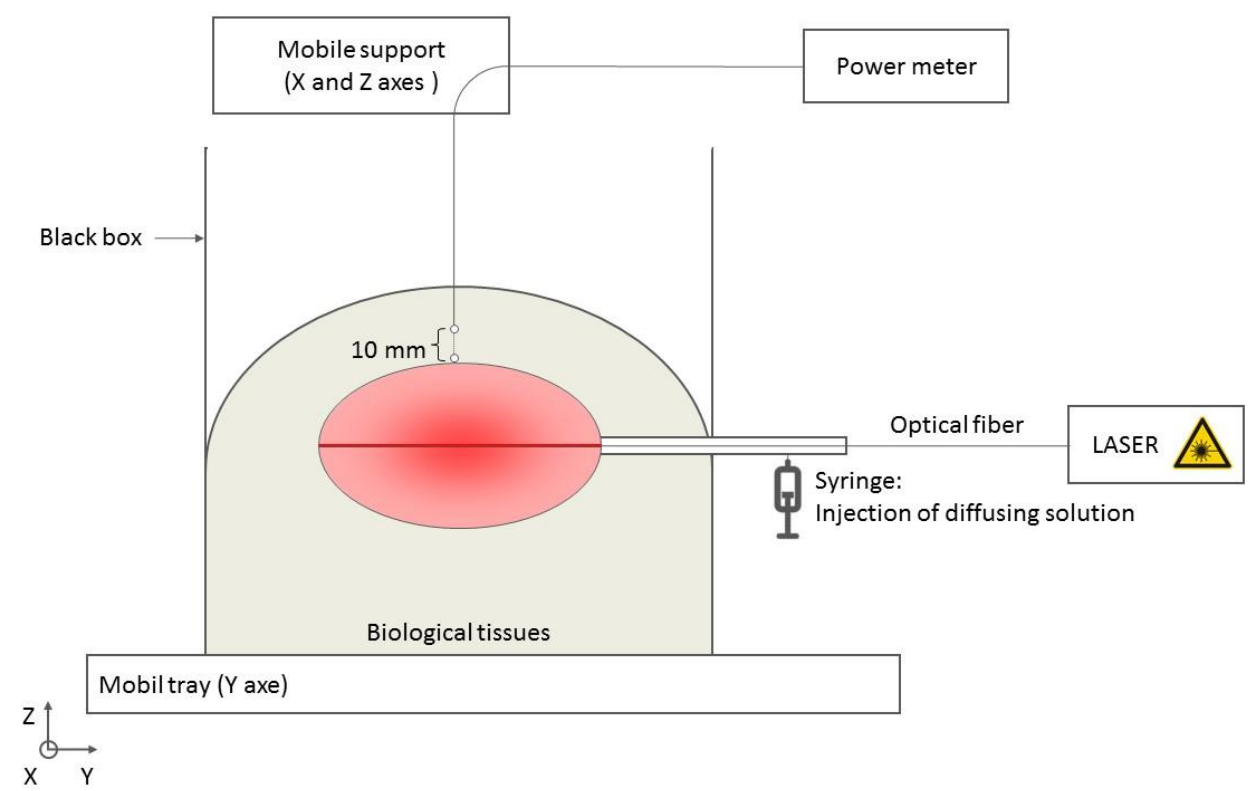

Figure 4. Experimental plan for fluence rate measurements of biological tissues: the device was placed inside a black box and surrounded by biological tissues. An isotropic probe was inserted into the biological tissues. Power measurements were performed at a distance of $10 \mathrm{~mm}$ from the balloon wall and with distance steps of $1 \mathrm{~mm}$. Fifteen different measurement

localizations were used to decrease the variability caused by tissue heterogeneity.

\section{Temperature measurements}

The temperature elevation was also investigated to evaluate the temperature elevation risk of surrounding brain tissues (figure 5). The balloon was filled with $500 \mathrm{~mL}$ of diffusing solution and then immersed in a hot-water bath adjusted to $37^{\circ} \mathrm{C}$. The LASER emitted a continuous power of $2 \mathrm{~W}$ for two hours. The temperature was measured with a thermographic camera. 

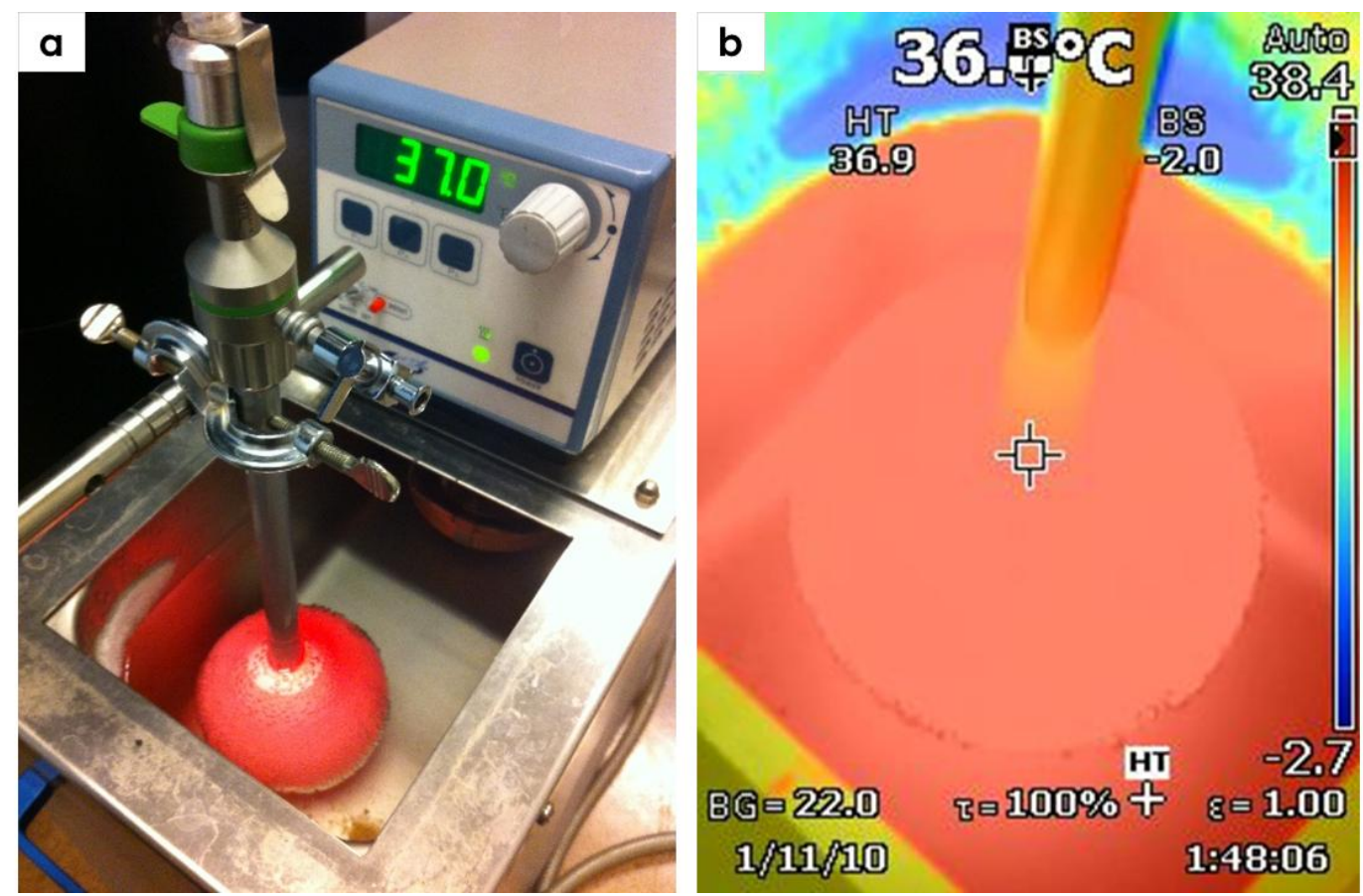

Figure 5 (a) Picture of the experimental set up of temperature measurement: the balloon was filled with $500 \mathrm{~mL}$ of diffusing solution and immersed in a hot-water bath adjusted to $37^{\circ} \mathrm{C}$. (b) Screenshot of the thermographic camera: the laser emitted a continuous power of $2 \mathrm{~W}$ for two hours.

\section{Results}

\section{Impact of the absence of PpIX in the biological tissue}

It is known that a PS might induce a change in optical coefficients according to its concentration in tissues. Therefore, we evaluated the impact of PpIX using the equation described by Vignion-Dewalle et al. [21], where PpIX accumulation does not affect the total reduced scattering coefficient. However, the absorption coefficient is the sum of the calf brain absorption $\mu_{a, \text { brain }}$ and the PpIX absorption $\mu_{a, P p I X}$ (equation 2).

$$
\mu_{a, \text { total }}=\mu_{a, \text { brain }}+\mu_{a, P p I X}
$$

where $\mu_{a, t o t a l}$ is the absorption coefficient due to the brain and PS absorption $\left(\mathrm{cm}^{-1}\right)$, $\mu_{a, \text { brain }}$ is the absorption coefficient of the calf brain $\left(\mathrm{cm}^{-1}\right)$, and $\mu_{a, P p I X}$ is the absorption coefficient of $\operatorname{PpIX}\left(\mathrm{cm}^{-1}\right)$.

We can express the PpIX absorption coefficient $\mu_{\mathrm{a}, \mathrm{PpIX}}$ according to the PpIX concentration $\mathrm{C}_{\mathrm{PpIX}}$ and its molar extinction coefficient $\varepsilon_{\mathrm{PpIX}}$ at the precise wavelength $\lambda$ (equation 3).

$$
\mu_{a, P p I X, \lambda}=\varepsilon_{P p I X, \lambda} \cdot C_{P p I X}
$$

where $\mu_{\mathrm{a}, \mathrm{Pp} I \mathrm{X}, \lambda}$ is the PpIX absorption coefficient at wavelength $\lambda\left(\mathrm{cm}^{-1}\right)$, 
$\mathrm{C}_{\mathrm{PpIX}}$ is the PpIX concentration (mol. $\mathrm{L}^{-1}$ ), and

$\varepsilon_{\mathrm{PpIX}, \lambda}$ is the molar extinction coefficient of the PpIX at wavelength $\lambda\left(\mathrm{L}_{\mathrm{mol}}{ }^{-1} \cdot \mathrm{cm}^{-1}\right)$

Thus, the PpIX absorption coefficient can be computed in brain tissues with high PpIX concentration at $635 \mathrm{~nm}$ (equation 4). In the study of Valdes et al. [22], a PpIX concentration of $4.523 \mu \mathrm{g} / \mathrm{mL}$ (approximately $8.039 \mathrm{~mol} . \mathrm{L}^{-1}$ ) was observed in GBM tissue with a high level of fluorescence and PpIX accumulation. The value of the PpIX molar extinction coefficient at $405 \mathrm{~nm}$ is known [23]. The PpIX absorption coefficients at $405 \mathrm{~nm}$ and $635 \mathrm{~nm}$ of a sample of an unknown concentration were determined from the absorption spectrum measured by the Research Center for Automatic Control of Nancy (CRAN).

$$
\mu_{a, P p I X, 635}=\frac{\mu_{a, P p I X, C R A N, 635}}{\mu_{a, P p I X, C R A N, 405}} \cdot \varepsilon_{P p I X, 405} \cdot C_{P p I X}
$$

where $\mu_{\mathrm{a}, \mathrm{PpIX}, 635}$ is the PpIX absorption coefficient at a wavelength of $635 \mathrm{~nm}\left(\mathrm{~cm}^{-1}\right)$, $\mu_{\mathrm{a}, \mathrm{PpIX}, \mathrm{CRAN}, 635}$ is the PpIX absorption coefficient of a sample of unknown concentration at a wavelength of $635 \mathrm{~nm}\left(\mathrm{~cm}^{-1}\right)$,

$\mu_{\mathrm{a}, \mathrm{PpIX}, \mathrm{CRAN}, 405}$ is the PpIX absorption coefficient of a sample of unknown concentration at a wavelength of $405 \mathrm{~nm}\left(\mathrm{~cm}^{-1}\right)$,

$\varepsilon_{\mathrm{PpIX}, 405}$ is the molar extinction coefficient of PpIX at a wavelength of $405 \mathrm{~nm}\left(\mathrm{~L}_{\mathrm{mol}}{ }^{-1} . \mathrm{cm}^{-1}\right)$, and

$\mathrm{C}_{\mathrm{PpIX}}$ is the PpIX concentration (mol. $\left.\mathrm{L}^{-1}\right)$.

From equation 4, the PpIX absorption coefficient in this condition and at $635 \mathrm{~nm}$ was thus estimated to be $0.0187 \mathrm{~cm}^{-1}$ in such a manner that the total absorption coefficient was only increased by $8.5 \%\left(\mu_{a, \text { total }}=0.2187 \mathrm{~cm}^{-1}\right)$.

\section{Fluence rate for different balloon states}

To convert power measurements into fluence rate values, the calibration factor $\mathrm{CF}_{\text {brain }}$ for the isotropic probe used in our experiments was computed using data provided by the Marijnissen and Star studies $[19,20]$ (equation 5).

$$
\Psi_{\text {media }}=\left(\mathrm{P}_{\text {measured }} \cdot \mathrm{CF}_{\text {air }}\right) \cdot \mathrm{F}_{\mathrm{n}} \cdot \mathrm{F}_{\mathrm{p}} \cdot \mathrm{F}_{\mathrm{b}} \cdot \mathrm{F}_{\mathrm{i}}
$$

with the following: $\mathrm{CF}_{\text {air }}=31721 \mathrm{~cm}^{-2}$

$\mathrm{F}_{\mathrm{n}}=1.55 \pm 0.05$ (dimensionless)

$\mathrm{F}_{\mathrm{p}}=1.03 \pm 0.02$ (dimensionless)

$\mathrm{F}_{\mathrm{b}}=1.0625 \pm 0.005$ (dimensionless)

$\mathrm{F}_{\mathrm{i}}=1.0237 \pm 0.07$ (dimensionless)

$\mathrm{CF}_{\text {brain }}=55083 \pm 6872 \mathrm{~cm}^{-2}$

In our conditions, the fluence rate values could be approximated with the following equation: 


$$
\Psi_{\text {media }}=\mathrm{P}_{\text {measured }} \cdot \mathrm{CF}_{\text {brain }}
$$

For a power of $2 \mathrm{~W}$ emitted by the $70 \mathrm{~mm}$ cylindrical diffuser, Figure 6 shows all fluence rate $\left(\mathrm{mW} / \mathrm{cm}^{2}\right)$ curves measured around the device for the different balloon volumes $(40 \mathrm{~mL}$ to $500 \mathrm{~mL})$.

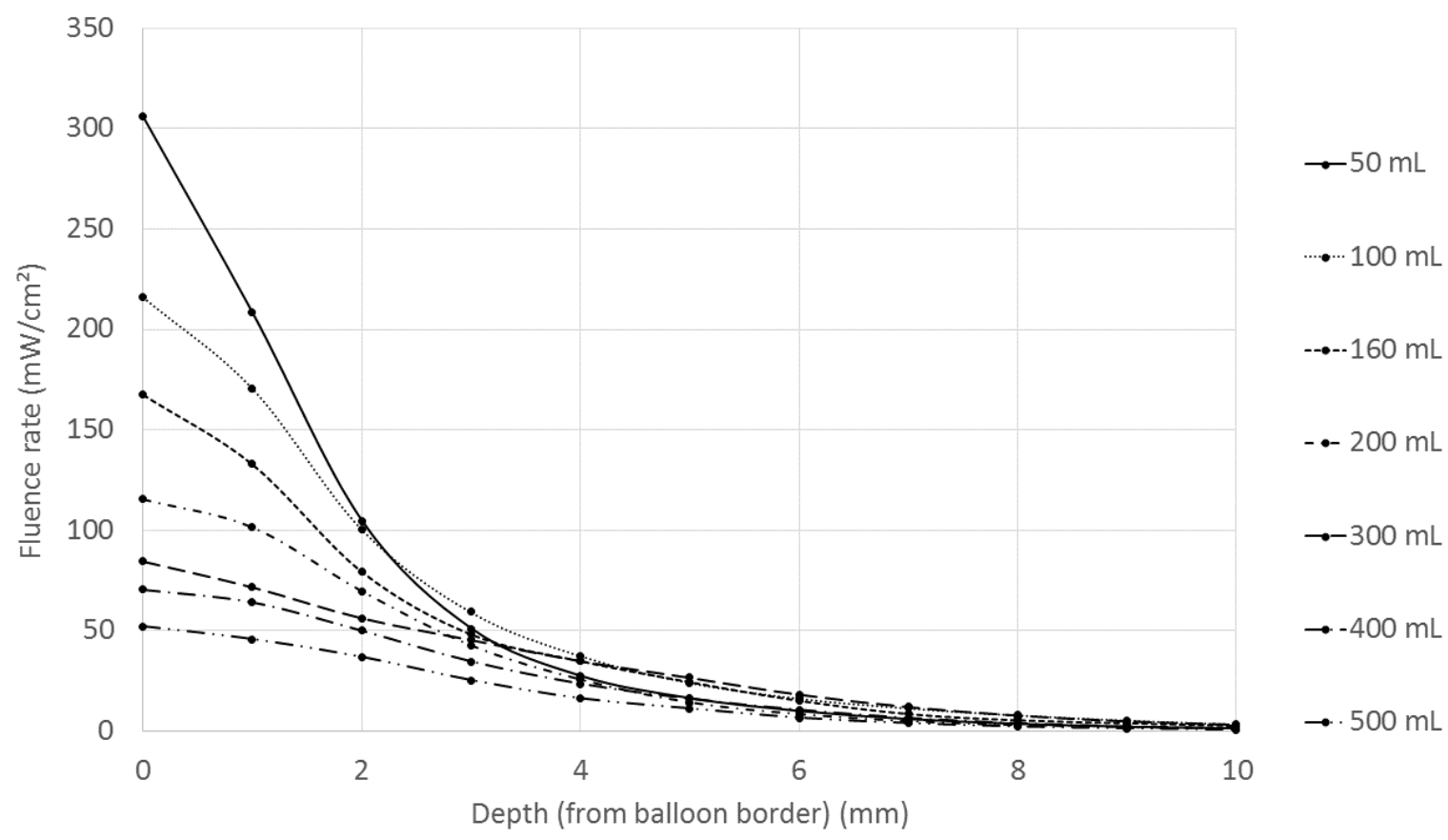

Figure 6. Sample of fluence rate $\left(\mathrm{mW} / \mathrm{cm}^{2}\right)$ curves measured in brain tissues at various distances from the balloon wall and for different balloon volumes

Then, fluence values were deduced from these fluence rate data. For a value of 200 $\mathrm{J} / \mathrm{cm}^{2}$ defined against the balloon wall, we obtained a fluence value close to $25 \mathrm{~J} / \mathrm{cm}^{2}$ at a 5 $\mathrm{mm}$ depth, independent of the balloon volume.

With this constraint, we computed the lighting duration to reach these fluence values for each balloon volume. A transfer function was defined to determine the lighting duration necessary to obtain a fluence value of $25 \mathrm{~J} / \mathrm{cm}^{2}$ [24-27] at a distance of $5 \mathrm{~mm}$ from the balloon wall depending on the volume of diffusing solution injected into the balloon (see figure 7). 


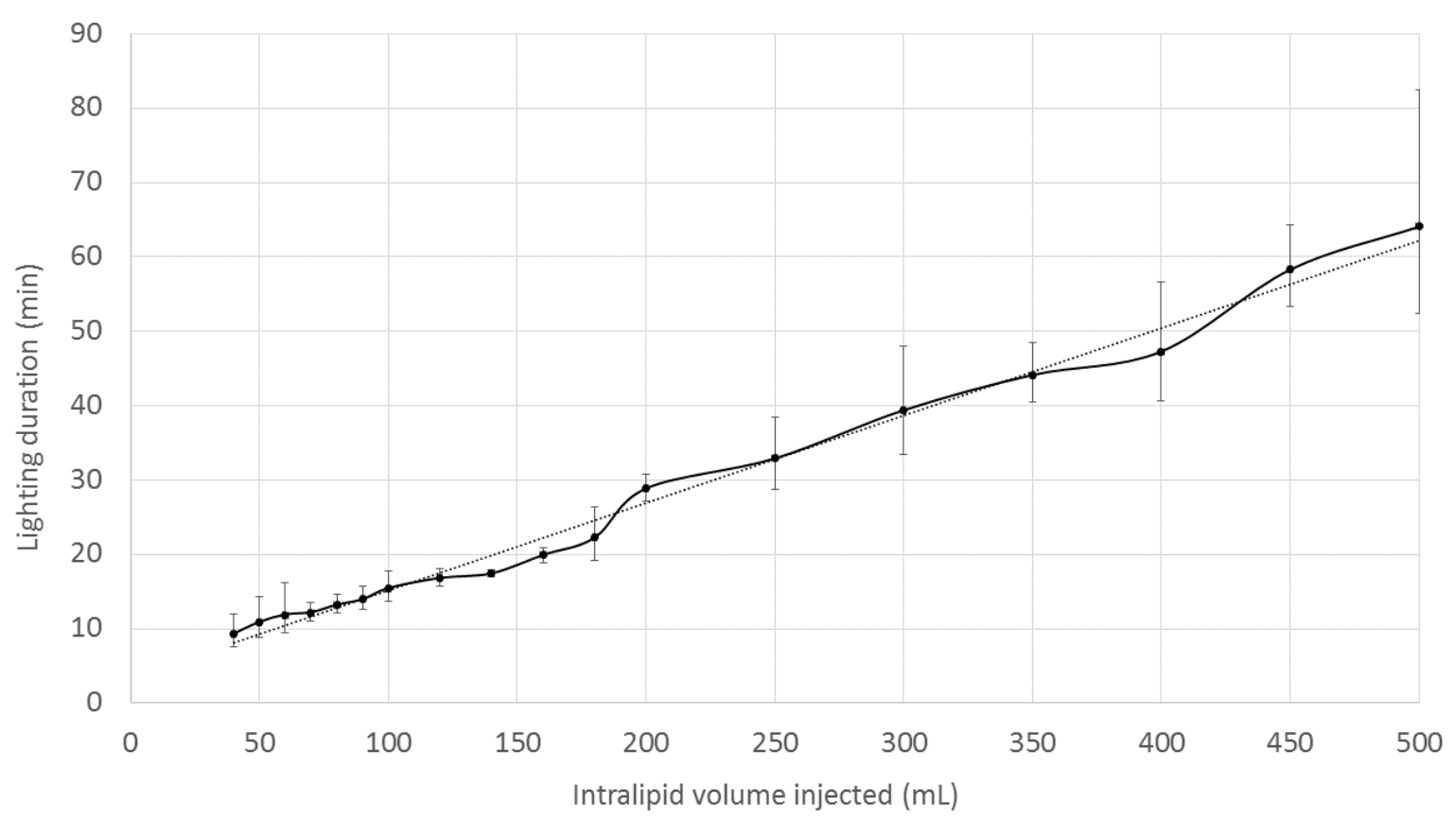

Figure 7. Transfer function of lighting duration (minutes) necessary to obtain a therapeutic fluence value of $25 \mathrm{~J} / \mathrm{cm}^{2}$ at a distance of $5 \mathrm{~mm}$ from the balloon wall depending on the volume of diffusing solution injected into the device

\section{Fluence rate and irradiance}

Monte-Carlo simulations were performed with the mcxyz Monte-Carlo program [28] to compare irradiance and fluence rate. The dimensions of the balloon in figure 8 correspond to a standard surgical cavity volume, i.e., $150 \mathrm{~mL}$ of diffusing solution injected into the device (approximately $150 \mathrm{~cm}^{3}$ ).
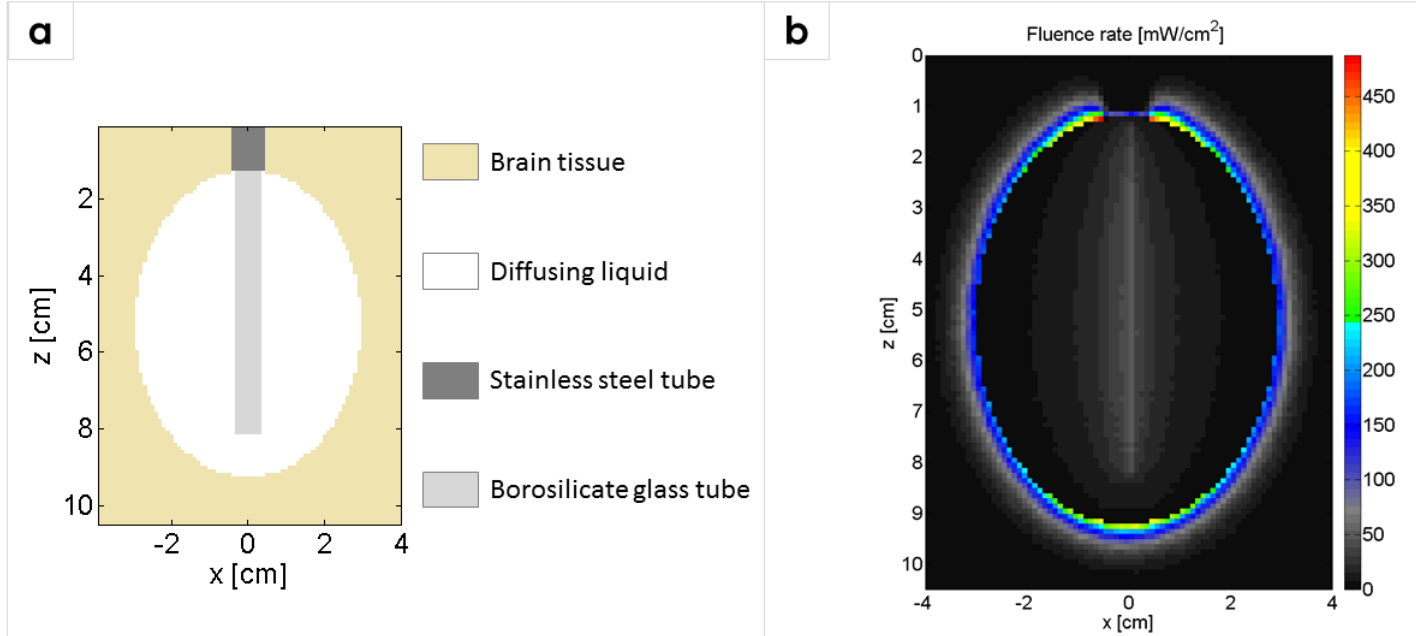

Figure 8 (a) Modeling of the device filled with $150 \mathrm{~mL}$ of diffusing solution. The 70 $\mathrm{mm}$ long cylindrical diffuser was located in the center of the borosilicate glass tube. (b) 
Results from the Monte-Carlo simulation: the fluence rate values obtained with the MonteCarlo simulation were close to the values measured in calf brains (Table 1).

Table 1: Comparison of fluence rates obtained from Monte-Carlo simulations and ex vivo measurements

\begin{tabular}{|c|c|c|c|}
\hline \multirow{2}{*}{$\begin{array}{l}\text { Volume of the } \\
\text { Balloon }(\mathrm{mL})\end{array}$} & \multicolumn{2}{|c|}{ Fluence rate at balloon border $\left(\mathrm{mW} / \mathrm{cm}^{2}\right)(\mathrm{SD})$} & \multirow[t]{2}{*}{ Error $(\%)$} \\
\hline & Monte-Carlo simulation & Ex vivo experiments & \\
\hline 50 & $280.82(18.47)$ & $305.79(72.86)$ & $8.89 \%$ \\
\hline 100 & $192.93(4.86)$ & $215.94(27.98)$ & $11.93 \%$ \\
\hline 150 & $175.45(21.13)$ & $179.52(7.02)$ & $2.13 \%$ \\
\hline 200 & $139.06(6.54)$ & $115.54(7.42)$ & $16.91 \%$ \\
\hline 250 & $115.30(6.42)$ & $101.36(14.74)$ & $14.44 \%$ \\
\hline Mean error (\%) & & & $10.90 \%$ \\
\hline
\end{tabular}

As an illustration of the difference between the irradiance and the fluence rate, figure 9 is a plot of the ratio of the fluence rate measured in the brain to the irradiance as a function of the depth for different balloon states.

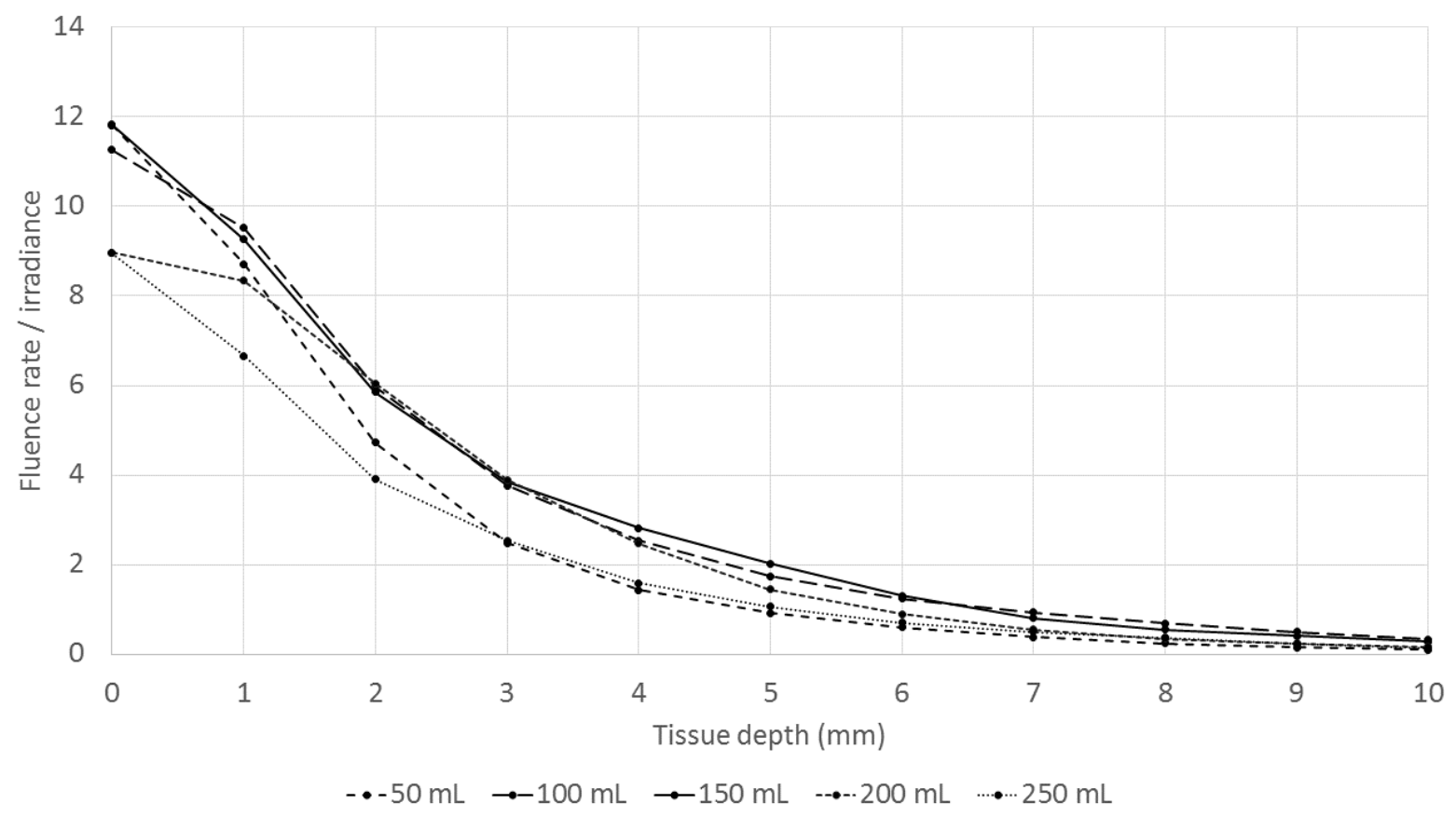

Figure 9: Plot of the ratio of the fluence rate measured in the brain to the irradiance as a function of the depth for different balloon states. 


\section{Consideration of temperature elevation}

Regarding the temperature measurements, we did not observe any temperature elevation during the experiments. Thus, the temperature elevation due to the device during treatment can be considered acceptable.

\section{Discussion}

Currently, despite interstitial PDT being more frequently referenced in the literature, it is mainly dedicated to recurrent or non-operable GBM. Because the population with recurrent GBM is non-homogeneous since patients have received multimodal treatment, highlighting PDT effects remains difficult. Additionally, this technique remains difficult to perform for a large $\operatorname{GBM}(>3.5 \mathrm{~cm})$.

In contrast to the situation for recurrent GBM, the management of a patient harboring newly diagnosed GBM must be in accordance with specific recommendations of standard of care [7]. Maximal EOR is thus expected when achievable and is mostly achieved by means of FGR. In this paper, we proposed a new device and associated method to easily incorporate intraoperative PDT into the standard surgical workflow. Indeed, the PDT effect on GBM is still not proven because of the poor reproducibility of the procedure from one center to another, the multi-modality treatment when considering only the recurrent GBM population and the low inclusion numbers in the past trials. The design of this new device aimed to simplify the PDT procedure for the neurosurgeon with a seamless methodology suitable for the standard surgical workflow that also limited the dosimetry issue during surgery. In such a manner, and thanks to a highly reproducible procedure, the effects of PDT should be better highlighted on a larger and more homogeneous population of only newly diagnosed GBM patients. The feasibility of delivering PDT for the treatment of newly diagnosed GBM is currently under evaluation in the INDYGO clinical trial (ClinicalTrials.gov identifier: NCT03048240).

Modeling with the function based on ex vivo experiments with the calf brains used in this study demonstrated that a value of $0.2 \mathrm{~cm}^{-1}$ appears to be a correct mean human brain absorption coefficient [3, 16, 17]. However, ex vivo experiments could not be performed after 5-ALA administration, and the PpIX metabolism was not able to be modeled. Thus, the impact of the absence of PpIX in the biological sample was evaluated prior to our experiments. Indeed, differences in the absorption coefficients with and without PpIX might lead to different fluence rates. Nevertheless, the PpIX absorption coefficient in this condition and at $635 \mathrm{~nm}$ is only responsible for an increase of $8.5 \%$ in the total absorption coefficient, and the impact of PpIX was thus considered negligible for our ex vivo experiments. Moreover, the tissue surrounding the balloon would have a lower PpIX concentration than resected tumor tissues and the corresponding PpIX impact should still be more modest, confirming our assumption that the ex vivo model used in this study was well suited for the characterization of our device. 
Concerning the precision of converting power measurements into fluence rate values, the calibration factor requires several constants for the refraction index of the medium surrounding the probe. This data strongly increases the calibration factor estimated in the air. The impact of the standard deviation of the calibration factor in the biological medium induces a standard deviation of 62 seconds for the illumination time (approximately $11 \%$ of the treatment duration for a mean cavity volume). This value remains low compared with the total illumination time needed to treat one patient. We observed a slight influence of calf brain oxidation on the power measurements that cannot be quantified. However, to minimize this variable, fresh specimens preserved by refrigeration were employed during our experiments.

The fluence rate values obtained from power measurements could appear high because of confusion with irradiance, which is more commonly reported. Indeed, the fluence rate $\left(\mathrm{W} / \mathrm{cm}^{2}\right)$ can be larger than the irradiance $\left(\mathrm{W} / \mathrm{cm}^{2}\right)$, especially in the brain, which is a highly scattering medium [29]. Monte-Carlo simulations achieved with the mcxyz Monte-Carlo program [28] confirmed this observation. In particular, with the device modeled in $3 \mathrm{D}$, the obtained fluence rate values were close to values measured in the ex vivo model. A mean error of $10.90 \%$ was obtained (see table 1) and remains satisfactory in regards to the assumption of a homogenous brain tissue used for Monte-Carlo and the slight difference in balloon shape that may occur in ex vivo experiments. Finally, this mean error remains in the range of error documented by the American Association of Physicists in Medicine, in which an error between 10 to $15 \%$ for that purpose is acceptable [30].

Potential adverse events, such as additional time needed to deliver PDT during surgery, positioning of the device and brain compression, were carefully considered during the design of the device.

Concerning additional time required to complete the procedure, neurosurgery experts have validated the average lighting duration achievable by the device for most of the surgical cavities observed in our neurosurgery department. Indeed, the additional surgery time of 30 to 45 minutes needed to deliver intraoperative PDT is considered to have limited risk for the patient. We thus obtained an operating time acceptable for the range of volumes injected. Several studies reported a fluence value of $25 \mathrm{~J} / \mathrm{cm}^{2}$ to study PDT effect with 5-ALA [24-26] on GBM cells [27], and a photodynamic effect is expected to be observed at least within 5 $\mathrm{mm}$ of the cavity margin. Additionally, the proposed protocol relies on $2 \mathrm{~W}$ of power being emitted by the $70 \mathrm{~mm}$ cylindrical diffuser. At this power, a slight heat increase occurs against the diffuser tip. However, this temperature increase is isolated from the brain by the borosilicate glass tube and by several centimeters of diffusing liquid. Thus, no potential temperature increase can occur inside a patient's brain during the PDT procedure.

Concerning location and brain compression during the procedure, the device was designed to be positioned as deflated inside the surgical cavity. Once the surgeon judged its position as correct, the balloon was inflated with the diffusing solution. The filling stopped when the balloon reached the borders of the cavity and was visually assessed with the surgical microscope. Additionally, the illumination protocol divides the total lighting duration into five illumination sequences, separated by two-minute off periods. To further secure the device 
during these two minutes, the surgeon slightly deflates the balloon to limit parenchymal compression that might create tissue ischemia or hematoma.

Although several studies have already reported intraoperative PDT, some experimental conditions have changed since the last trial, and more particularly, the PS administered to the patient has changed. In the last clinical trial in 2007 [13, 14], the drug administered was Photofrin $^{\circledR}$ (Pinnacle Biologics ${ }^{\mathrm{TM}}$, Chicago, IL, United States). This first-generation PS had some disadvantages, including low absorbance at $630 \mathrm{~nm}$ and poor selectivity for tumor cells, leading to the necrosis of healthy tissues [31]. With the new generation of PSs, the selectivity and reactivity have been improved. In the surgical procedure investigated, we administered the precursor 5-ALA (Gliolan-Medac, Germany) to the patient, which induces a strong concentration of PpIX in tumor cells [32, 33]. With the application of our device early after FGR, improvement in the local control of direct PDT effects and an immune response with indirect PDT effects are expected.

\section{Conclusion}

In this study, we present a new device for intraoperative PDT dedicated to GBM treatment. An ex vivo study was performed, and we introduced a transfer function. This tool enables us to determine the lighting duration according to the surgery cavity size in order to achieve a therapeutic fluence value at a $5 \mathrm{~mm}$ depth within surrounding tissues. A methodology was described to convert power measurements to fluence rate values with the help of a sensor- and tissue-dependent calibration factor. The fluence rate in tissues around the device was estimated with ex vivo experiments. No thermal elevation was observed during a two-hour temperature test, and the PpIX impact on brain optical properties can be neglected. Several Monte-Carlo simulations were performed to confirm the fluence rate values measured in calf brains. A feasibility study of intraoperative PDT for newly diagnosed GBM with this device is currently running in our neurosurgery department (clinical trial: NCT03048240).

\section{Conflict of interest: None.}

Executive summary:

PDT for GBM treatment:

- Due to relapse in resection margins, local and selective therapy is required to treat glioblastoma (GBM).

- Photodynamic therapy (PDT) is an encouraging additional therapy to delay glioblastoma relapse.

- 5-aminolevulinic acid (5-ALA) is already used clinically as a contract agent in the fluorescence-guided resection (FGR) technique.

- PDT can easily be integrated in standard care and applied early after FGR. 
Characterization of a new device dedicated to intracavitary PDT:

- The device described in this publication, which is composed of an inflatable balloon filled with diffusing liquid, enables the illumination of both small and large resection cavities.

- The device was characterized using ex-vivo experiments: calf brains were used to mimic the optical properties of the human brain.

- Power measurements were performed around the balloon inside brain tissues at a depth of $10 \mathrm{~mm}$ for different volumes of diffusing liquid ( $40 \mathrm{~mL}$ to $500 \mathrm{~mL}$ ).

- Power measurements were converted into fluence rate values using Marijnissen and Star methodology.

- Fluence rate values were validated using Monte-Carlo simulations.

A new tool for intraoperative PDT:

- From these fluence rate values, an innovative transfer function was designed to determine the lighting duration necessary to deposit a fluence value of $25 \mathrm{~J} / \mathrm{cm}^{2}$ at a 5 mm depth independent of the cavity size.

- The additional 30 to 45 minutes of surgery time needed to deliver intraoperative PDT is considered to have limited risks for the patient.

- Other safety tests, including temperature measurements, were performed to ensure the device. No temperature increase can occur during the PDT procedure.

- High concentrations of protoporphyrin IX are responsible for the $8.5 \%$ increase in the total absorption coefficient in the human brain at $635 \mathrm{~nm}$.

Conclusions:

- A safety evaluation of the device and PDT procedure is currently under way in the INDYGO clinical trial (ClinicalTrials.gov identifier: NCT03048240).

- With application of the device early after FGR, improvement of the local control of direct PDT effects and immune responses with indirect PDT effects are expected.

1. Stupp R HM, Mason Wp, Et Al. Effects of radiotherapy with concomitant and adjuvant temozolomide versus radiotherapy alone on survival in glioblastoma in a randomised phase III study: 5-year analysis of the EORTC-NCIC trial. Lancet Oncol doi:10.1016/S1470-2045(09)70025-7 8 (2009).

2. Agostinis P, Berg K, Cengel KA et al. Photodynamic therapy of cancer: an update. CA: a cancer journal for clinicians 61(4), 250-281 (2011).

3. Beck TJ, Kreth FW, Beyer W et al. Interstitial photodynamic therapy of nonresectable malignant glioma recurrences using 5-aminolevulinic acid induced protoporphyrin IX. Lasers in Surgery and Medicine 39(5), 386-393 (2007).

4. Johansson A, Faber F, Kniebuhler G et al. Protoporphyrin IX fluorescence and photobleaching during interstitial photodynamic therapy of malignant gliomas for early treatment prognosis. Lasers in Surgery and Medicine 45(4), 225-234 (2013). 
5. Olzowy B, Hundt CS, Stocker S, Bise K, Reulen HJ, Stummer W. Photoirradiation therapy of experimental malignant glioma with 5-aminolevulinic acid. J Neurosurg 97(4), 970-976 (2002).

6. Stylli SS, Kaye AH, Macgregor L, Howes M, Rajendra P. Photodynamic therapy of high grade glioma - long term survival. Journal of Clinical Neuroscience 12(4), 389398 (2005).

7. Stupp R, Brada M, Van Den Bent MJ, Tonn JC, Pentheroudakis G, Group EGW. High-grade glioma: ESMO Clinical Practice Guidelines for diagnosis, treatment and follow-up. Ann Oncol 25 Suppl 3 iii93-101 (2014).

* 8. Leroy HA, Vermandel M, Lejeune JP, Mordon S, Reyns N. Fluorescence guided resection and glioblastoma in 2015: A review. Lasers in Surgery and Medicine 47(5), 441-451 (2015).

Indicates that $85 \%$ of cases will have recurrence in the surgical margins

9. Petrecca K, Guiot MC, Panet-Raymond V, Souhami L. Failure pattern following complete resection plus radiotherapy and temozolomide is at the resection margin in patients with glioblastoma. J Neurooncol 111(1), 19-23 (2013).

10. Pavlov V, Page P, Abi-Lahoud G et al. Combining intraoperative carmustine wafers and Stupp regimen in multimodal first-line treatment of primary glioblastomas. $\mathrm{Br} J$ Neurosurg 29(4), 524-531 (2015).

11. Wilson BC, Muller PJ, Yanch JC. Instrumentation and light dosimetry for intraoperative photodynamic therapy (PDT) of malignant brain tumours. Physics in Medecine and Biology 31(2), 125-133 (1986).

12. Dwyer PJ, White WM, Fabian RL, Anderson RR. Optical integrating balloon device for photodynamic therapy. Lasers in Surgery and Medicine 26(1), 58-66 (2000).

13. Moseley $\mathrm{H}$, Mclean $\mathrm{C}$, Hockaday $\mathrm{S}$, Eljamel $\mathrm{S}$. In vitro light distributions from intracranial PDT balloons. Photodiagnosis Photodyn Ther 4(3), 213-220 (2007).

14. Eljamel MS, Goodman C, Moseley H. ALA and Photofrin fluorescence-guided resection and repetitive PDT in glioblastoma multiforme: a single centre Phase III randomised controlled trial. Lasers in medical science 23(4), 361-367 (2008).

15. Dupont C, Betrouni N, Tylcz J-B et al. A novel device for intraoperative photodynamic therapy dedicated to glioblastoma treatment. 36th Annual Conference of the American Society for Laser Medicine and Surgery 48(27), 54 (2016).

16. Cheong WF, Prahl SA, Welch AJ. A review of the optical properties of biological tissues. IEEE Journal of Quantum Electronics 26(12), 2166-2185 (1990).

* 17. Jacques SL. Optical properties of biological tissues: a review. Physics in Medecine and Biology 58(11), R37-61 (2013).

Review of light propagation modeling

18. Kostron H, Kaneko S, Stepp H, Eljamel S. CHAPTER 20 Photodynamic Medicine in Neurosurgery: Biochemical, Technical and Clinical Aspects. In: Photodynamic Medicine: From Bench to Clinic, (Ed.^(Eds).The Royal Society of Chemistry 353403 (2016).

* 19. Marijnissen JP, Star WM. Calibration of isotropic light dosimetry probes based on scattering bulbs in clear media. Phys Med Biol 41(7), 1191-1208 (1996).

* 20. Marijnissen JP, Star WM. Performance of isotropic light dosimetry probes based on scattering bulbs in turbid media. Phys Med Biol 47(12), 2049-2058 (2002).

Fundamental work allowing the conversion of power measurement into fluence rate values that is promoted by the American Association of Physicists in Medicine 
21. Vignion-Dewalle AS, Betrouni N, Tylcz JB, Vermandel M, Mortier L, Mordon S. Comparison of three light doses in the photodynamic treatment of actinic keratosis using mathematical modeling. Journal of biomedical optics 20(5), 58001 (2015).

* 22. Valdes PA, Kim A, Brantsch M et al. delta-aminolevulinic acid-induced protoporphyrin IX concentration correlates with histopathologic markers of malignancy in human gliomas: the need for quantitative fluorescence-guided resection to identify regions of increasing malignancy. Neuro Oncology 13(8), 846-856 (2011).

Provides PpIX concentration measurements in different GBM tissue types

23. Natarajan P, Raja C. Studies on interpolymer self-organisation behaviour of protoporphyrin IX bound poly(carboxylic acid)s with complimentary polymers by means of fluorescence techniques. European Polymer Journal 40(10), 2291-2303 (2004).

24. Hirschberg $\mathrm{H}$, Sun $\mathrm{CH}$, Krasieva T, Madsen SJ. Effects of ALA-mediated photodynamic therapy on the invasiveness of human glioma cells. Lasers Surg Med 38(10), 939-945 (2006).

25. Kamoshima Y, Terasaka S, Iwasaki Y. Photodynamic therapy mediated with 5aminolevulinic acid for C6 glioma spheroids. Hokkaido Igaku Zasshi 83(3), 167-173 (2008).

26. Zelenkov $\mathrm{P}$, Baumgartner $\mathrm{R}$, Bise $\mathrm{K}$ et al. Acute morphological sequelae of photodynamic therapy with 5-aminolevulinic acid in the C6 spheroid model. $J$ Neurooncol 82(1), 49-60 (2007).

27. Tirapelli LF, Morgueti M, Da Cunha Tirapelli DP et al. Apoptosis in glioma cells treated with PDT. Photomedicine and laser surgery 29(5), 305-309 (2011).

28. Jacques S, Li T, Prahl S. mcxyz. 2017 (2013).

** 29. Welch AJ, Van Gemert MJC, Star WM. Definitions and Overview of Tissue Optics. In: Optical-Thermal Response of Laser-Irradiated Tissue, Welch AJ,Van Gemert MJC (Ed. ${ }^{\wedge}$ (Eds).Springer Netherlands Dordrecht 27-64 (2011).

Excellent chapter clearly explaining the difference between irradiance and fluence rate

30. Hetzel FW, Brahmavar SM, Chen Q et al. Photodynamic Therapy Dosimetry. (978-1888340-51-8), (2005).

31. Juzeniene A, Peng Q, Moan J. Milestones in the development of photodynamic therapy and fluorescence diagnosis. Photochem Photobiol Sci 6(12), 1234-1245 (2007).

32. Tetard MC, Vermandel M, Mordon S, Lejeune JP, Reyns N. Experimental use of Photodynamic Therapy in high grade gliomas: a review focused on 5-aminolevulinic acid. Photodiagnosis Photodyn Ther doi:10.1016/j.pdpdt.2014.04.004 19 (2014).

33. Krammer B, Plaetzer K. ALA and its clinical impact, from bench to bedside. Photochem Photobiol Sci 7(3), 283-289 (2008). 